\title{
Nonclassical Solutions of Fully Nonlinear Elliptic Equations
}

\author{
Nikolai Nadirashvili;, Serge Vlăduț ${ }^{\dagger}$
}

\begin{abstract}
We prove the existence of non-smooth solutions to fully nonlinear elliptic equations.
\end{abstract}

\section{Introduction}

In this paper we study the regularity of solutions of fully nonlinear elliptic equations of the form

$$
F\left(D^{2} u\right)=0
$$

defined in a domain of $\mathbf{R}^{n}$. Here $D^{2} u$ denotes the Hessian of the function $u$. We assume that $F$ is uniformly elliptic, i.e. there exists a constant $\Lambda \geq 1$ (called an ellipticity constant) such that

$$
\Lambda^{-1}|\xi|^{2} \leq F_{u_{i j}} \xi_{i} \xi_{j} \leq \Lambda|\xi|^{2}, \forall \xi \in \mathbf{R}^{n} .
$$

Here, $u_{i j}$ denotes the partial derivative $\partial^{2} u / \partial x_{i} \partial x_{j}$. A function $u$ is called a classical solution of (1) if $u \in C^{2}(\Omega)$ and $u$ satisfies (1). Actually, any classical solution of (1) is a smooth $\left(C^{\alpha+3}\right)$ solution, provided that $F$ is a smooth $\left(C^{\alpha}\right)$ function of its arguments.

Consider the following Dirichlet problem

$$
\begin{cases}F\left(D^{2} u\right)=0 & \text { in } \Omega \\ u=\varphi & \text { on } \partial \Omega\end{cases}
$$

where $\Omega \subset \mathbf{R}^{n}$ is a bounded domain with smooth boundary $\partial \Omega$ and $\varphi$ is a continuous function on $\partial \Omega$.

It is not difficult to prove that problem (3) has no more than one classical solution (see e.g. [GT]). The basic problem is the existence of such classical solutions. Although the first systematic study of the Dirichlet problem for fully

*LATP, CMI, 39, rue F. Joliot-Curie, 13453 Marseille FRANCE, nicolas@cmi.univ-mrs.fr

${ }^{\dagger}$ IML, Luminy, case 907, 13288 Marseille Cedex FRANCE, vladut@iml.univ-mrs.fr 
nonlinear equations was done by Bernstein at the beginning of the 20-th century (see [GT]), the first complete result didn't appear until 1953, when Nirenberg proved the existence of a classical solution to problem (3) in dimension $n=2$ ([N]). For $n \geq 3$, the problem of the existence of classical solutions to Dirichlet problem (3) remained open.

In order to get a solution to the problem (3) one can try to extend the notion of the classical solution of the equation (1). That was done recently: CrandallLions and Evans developed the concept of viscosity (weak) solutions of the fully nonlinear elliptic equations. As a characteristic property for such extension can be taken the maximum principle in the following form:

Let $u_{1}, u_{2}$ be two solutions of the following equations, $F\left(D^{2} u_{1}\right)=f_{1}$ in $\Omega$ and $F\left(D^{2} u_{2}\right)=f_{2}$ in $\Omega$. Then for any subdomain $G \subset \Omega$ the inequalities $f_{1} \leq f_{2}\left(f_{1} \geq f_{2}\right)$ in $G$ and $u_{1} \geq u_{2}\left(u_{1} \leq u_{2}\right)$ on $\partial G$ imply the inequality $u_{1} \geq u_{2}\left(u_{1} \leq u_{2}\right)$ in $G$.

Such maximum principle holds for $C^{2}$ functions $u_{1}, u_{2}$. We call a continuous function $u_{1}$ a viscosity solution of $F\left(D^{2} u_{1}\right)=f_{1}$ if the above maximum principle holds for $u_{1}$ and all $C^{2}$-functions $u_{2}$.

It is possible to prove the existence of a viscosity solution to the Dirichlet problem (3) and Jensen's theorem says that the viscosity solution of the problem (3) is unique. For more details see [CC], [CIL].

There are important classes of the fully nonlinear Dirichlet problems for which the viscosity solution is in fact a classical one, e.g., due to Krylov-Evans regularity theory, in the case when the function $F$ is convex, (see $[\mathrm{CC}],[\mathrm{K}]$ ). However, for the general $F$ the problem of the coincidence of viscosity solutions with the classical remained open.

The central result of this paper is the existence of nonclassical viscosity solution of (1) in the dimension 12. More precisely we prove

Theorem. The function

$$
w(x):=\frac{\operatorname{Re}\left(\omega_{1} \omega_{2} \omega_{3}\right)}{|x|},
$$

where $\omega_{i} \in \mathbf{H}, \quad i=1,2,3$, are Hamiltonian quaternions, $x=\left(\omega_{1}, \omega_{2}, \omega_{3}\right) \in$ $\mathbf{H}^{3}=\mathbf{R}^{12}$ is a viscosity solution in $\mathbf{R}^{12}$ of a uniformly elliptic equation (1) with a smooth $F$.

One can find the explicit expression for $w$ in the coordinates of $\mathbf{R}^{12}$ in Sections 3 and 4 . The elliptic operator $F$ will be defined in a constructive way in Section 2, and its ellipticity constant $\Lambda<10^{8}$.

As an immediate consequence of the theorem we have

Corollary. Let $\Omega \subset \mathbf{R}^{12}$ be the unit ball and $\varphi=w$ on $\partial \Omega$. Then there exists a smooth uniformly elliptic $F$ such that the Dirichlet problem (2) has no classical solution. 
Homogeneous order 2 function $w$ is smooth in $\mathbf{R}^{12} \backslash\{0\}$ and has discontinuous second derivatives at 0 . It is interesting to notice that the set of homogeneous order $\alpha \in \mathbf{R}$ solutions of (1) in $\mathbf{R}^{n} \backslash\{0\}$ for $\alpha \neq 2$ has a simple structure: each such solution of (1) has to be also a solution of a linear elliptic equation with constant coefficients, [NY].

The question on the minimal dimension $n$ for which there exist nontrivial homogeneous order 2 solutions of (1) remains open. We notice that from the result of Alexsandrov $[\mathrm{A}]$ it follows that any homogeneous order 2 solution of the equation (1) in $\mathbf{R}^{3}$ with a real analytic $F$ should be a quadratic polynomial. For a smooth and less regular $F$ similar results in the dimension 3 one can find in $[\mathrm{HNY}]$.

Acknowledgements. The authors would like to thank S. Kuksin and Y.Yuan for very useful discussions as well as the anonymous referee for his very pertinent remarks.

\section{The Hessian Problem}

\section{1}

Let $w$ be a homogeneous function of order 2, defined on $\mathbf{R}^{n}$ and smooth in $\mathbf{R}^{n} \backslash\{0\}$. Then the Hessian of $w$ is homogeneous of order 0, and defines a map

$$
H: S^{n-1} \rightarrow D^{2} w \in Q,
$$

where $Q$ denotes the space of quadratic forms on $\mathbf{R}^{n}$, which we will sometimes identify as symmetric $n \times n$ matrices, $Q \simeq S^{n \times n}$. The inner product of $a, b \in Q$ is given by $a \cdot b=\operatorname{trace}(a b)$. We say that $w$ satisfies property $(\mathrm{H})(w$ is a solution of the Hessian Problem) if the following holds:

(H) 1). The map $H: S^{n-1} \rightarrow Q$ is a smooth embedding.

2). There exists a constant $M \geq 1$ such that for any two points $a, b \in$ $H\left(S^{n-1}\right), a \neq b$, if $\mu_{1} \geq \cdots \geq \mu_{n}$ denote the eigenvalues of the quadratic form $a-b$, then

$$
1 / M<-\mu_{1} / \mu_{n}<M .
$$

Main Lemma. If function $w$ satisfies hypotheses $(\mathrm{H})$ then $w$ is a viscosity solution in $\mathbf{R}^{n}$ of a uniformly elliptic equation (1) . 


\section{2}

Let us choose in the space

$Q$ an orthonormal coordinate system $z_{1}, \ldots, z_{k}, s, k=\frac{n(n+1)}{2}-1$ such that $\sqrt{n} s$ is the trace. Let $\pi: Q \rightarrow Z$ be the orthogonal projection of $Q$ onto the $z$-space. For $\lambda \geq 1$, we denote by $K_{\lambda}$ the cone

$$
K_{\lambda}=\{a \in Q: \text { there exists } C>0 \text { s. t. the eigenvalues of } a \in[C / \lambda, C \lambda]\} .
$$

Notice, that inequalities (2) mean that the eigenvalues of $\nabla F$ are on the segment $\left[\Lambda^{-1}, \Lambda\right]$. In particular (2) implies the inclusion $\nabla F \in K_{\Lambda}$. Since $0 \notin K_{\Lambda}$ it follows in particular that $F^{(-1)}(0) \in C^{\infty}$.

Since on $Q$ the maximal eigenvalue of a quadratic form is a convex function and the minimal eigenvalue is a concave function it follows that $K_{\Lambda}$ is a convex cone.

Let $K_{\lambda}^{*}$ denote the adjoint cone of $K_{\lambda}$, that is,

$$
K_{\lambda}^{*}=\left\{b \in Q: b \cdot c \geq 0 \text { for all } c \in K_{\lambda}\right\} .
$$

As an adjoint to a convex cone the cone $K_{\lambda}^{*}$ is a convex cone itself.

Set $L_{\lambda}=Q \backslash\left(K_{\lambda}^{*} \cup-K_{\lambda}^{*}\right)$. Notice that $a \in L_{\lambda}$ is equivalent to $a \cdot b=0$ for some $b \in K_{\lambda}$, i.e., $L_{\lambda}$ is a union of all hyper-planes in $Q$ with normals in $K_{\lambda}$. Since the quadratic forms of $K_{\lambda}$ are positively defined it follows that the vector $I \in K_{\lambda}^{*}$. Let $K \subset Q$ be a cone with a smooth strictly convex base such that $K_{2 \lambda} \subset K \subset K_{\lambda}$. Let $e_{1}, \ldots e_{k}, I$ be an orthonormal basis of $Q$ corresponding to the coordinates $z_{1}, \ldots, z_{k}, s$. Then any matrix $b \in Q$ can be written as

$$
b=s I+\sum_{j=1}^{k} z_{j} e_{j} .
$$

Now define

$$
x(z):=\inf \left\{c:(a+c I) \in K^{*}\right\}
$$

for $a:=\sum_{j=1}^{k} z_{j} e_{j}$. The graph of the function $s=x(z)$ represents the boundary of the cone $K$. Clearly $x(\cdot)$ is Lipshitz, convex, homogenous, smooth outside the origin and $x(0)=0$. By a simple computation we get that $|\nabla x|<\sqrt{n}$.

Let $G \subset Q$ be a set. We say that $G$ satisfies the $\lambda$-cone condition if for any two points $a, b \in G$, the matrix $a-b \in L_{\lambda}$.

Lemma. Let $\Sigma \subset Q$ be a smooth compact $(n-1)$-dimensional manifold. Assume that $\Sigma$ satisfies the $\lambda$-cone condition. Then there exists a smooth function $F$ on $Q$ such that $F(\Sigma)=0$, and which satisfies the inequality (2) with the ellipticity constant $\Lambda<4 \lambda^{2} \sqrt{n}$.

Proof of the lemma. Set $\sigma=\pi(\Sigma)$. We prove that $\Sigma$ is a graph of a Lipschitz continuous function,

$$
\Sigma=\{z \in \sigma: s=g(z)\}
$$


Let $a, \hat{a} \in \Sigma, a=s I+\sum_{j=1}^{k} z_{j} e_{j}, \hat{a}=\hat{s} I+\sum_{j=1}^{k} \hat{z}_{j} e_{j}$. Since $a-\hat{a} \in L_{\lambda}$, we have $-x(z-\hat{z}) \leq \hat{s}-s \leq x(z-\hat{z})$. Since $x(0)=0, g(z):=s$ is single-valued. Also $|g(z)-g(\hat{z})|=|s-\hat{s}| \leq|x(z-\hat{z})| \leq C|z-\hat{z}|$.

Hence, since $\Sigma$ is a smooth surface $g$ is a smooth function and $\sigma$ is a smooth surface as well.

Let $G_{k}^{m}$ be the Grassmannian manifold of $m$-dimensional subspaces of the $k$-dimensional subspace $z$ of $Q$. Let $l \in G_{k}^{n-1}$ and $t: l \rightarrow s$ be a linear function on $l$, such that the graph of $t$ satisfies the $\lambda$-cone condition. All such linear functions $t$ defined on all $l \in G_{k}^{n-1}$ we denote by $\tau$. Let $t \in \tau$ defined on $l \in G_{k}^{n-1}$ be such that $|\nabla t| \neq 0$. Then there exists a constant $c^{\prime}>1$ such that $c^{\prime} t \leq x$ on $l$ and there is a point $z^{\prime} \in l,\left|z^{\prime}\right|=1$ with $c^{\prime} t\left(z^{\prime}\right)=x\left(z^{\prime}\right)$. Since $K^{*}$ is a strictly convex cone the vector $z^{\prime}$ is unique. Denote

$$
\eta(t)=\{z \in l, t(z)=0\} .
$$

Then $\eta(t) \perp \nabla t$. Since $\eta(t) \subset Z$ and $\eta(t)$ is tangent to the cone $K^{*}$ at $z^{\prime}, x\left(z^{\prime}\right)$ it follows that $\eta(t) \perp \nabla x\left(z^{\prime}\right)$.

Let $\theta$ be a smooth function defined on $[1, \infty)$ such that $0 \leq \theta \leq 1, \theta=1$ on $[1, A], \theta=0$ on $[2 A, \infty)$, where a sufficiently large constant $\bar{A}$ will be chosen later. Set

$$
\nu(t)=\theta\left(c^{\prime}\right) \nabla x\left(z^{\prime}\right)+\left(1-\theta\left(c^{\prime}\right)\right) \nabla t .
$$

For $z \in \sigma$ we denote by $l(z) \in G_{k}^{(n-1)}$ the tangent subspace to $\sigma$ at $z$. Let $t_{z}(x), x \in l(z)$, be the differential of $g$ at $z$.

Let $z \in \sigma$. Denote by $\Psi(z)(n-1)$-dimensional subspace spanned by $\eta\left(t_{z}\right)$ and $\nu\left(t_{z}\right)$, if $\nabla t_{z} \neq 0$. If $\nabla t_{z}=0$ we set $\Psi(z)=l$. Thus we defined a smooth map

$$
\Psi: \sigma \rightarrow G_{k}^{n-1} \text {. }
$$

There exists $\gamma \subset Z$ a closed neighborhood of $\sigma$ such that $\gamma$ is diffeomorphic to $\sigma \times B$, where $B$ is the $(k-n+1)$-dimensional disk. We define a projection $\gamma \rightarrow \sigma$ such that the fiber $p^{-1}(z)$ is orthogonal to $\Psi(z)$ at $z \in \sigma$. Since $\left(\nabla x\left(z^{\prime}\right), z^{\prime}\right)>0$ the fiber $p^{-1}(z)$ is transversal to $\sigma$ at $z$. We extend the function $g$ to $\gamma$ by $g(y)=g(p(y))$. Let $\Gamma$ be the graph of $g$ over $\gamma$. Let $z \in \sigma$ and $d g(z)$ be the differential of $g$ over $\gamma$. For sufficiently large constant $A$ the following alternative holds: either $|\nabla g(z)|$ is sufficiently small, or the graph of $c^{\prime} d g(z)$ is tangent to the cone $K^{*}$. In both cases the graph of $d g(z)$ satisfies $2 \lambda$-cone condition. Since $g \in C^{1}(Z)$ and along the fibers the function $g$ is a constant, we may assume the neighborhood $\gamma$ to be sufficiently small so that $\Gamma$ satisfies the $\lambda$-cone condition.

Since $K_{2 \lambda} \subset K g \in C^{1}(\sigma)$ the function $g$ has an extension $\widetilde{g}$ from the set $\gamma$ to $\mathbf{R}^{k}$ such that $\widetilde{g}$ is a Lipschitz function and the graph of $\widetilde{g}$ satisfies the $2 \lambda$-cone condition. One can define such an extension $\widetilde{g}$ simply by the formula

$$
\widetilde{g}(z):=\inf _{w \in \gamma}\{g(w)+x(z-w)\}
$$


To demonstrate that this formula works let $(z, \tilde{g}(z))$, $(\hat{z}, \tilde{g}(\hat{z}))$ lie on the graph $\tilde{g}$. We must show

$$
-x(z-\hat{z}) \leq \tilde{g}(z)-\tilde{g}(\hat{z}) \leq x(z-\hat{z}) .
$$

Now

$$
\tilde{g}(\hat{z})=g(w)+x(\hat{z}-w)
$$

for some $w \in \gamma$. Thus

$$
\tilde{g}(z)-\tilde{g}(\hat{z}) \leq g(w(\hat{z}))+x(z-w(\hat{z}))-(g(w(\hat{z}))+x(\hat{z}-w(\hat{z}))) \leq x(z-\hat{z}),
$$

since $x(a+b) \leq x(a)+x(b)$, as $x(\cdot)$ is convex and homogenous. Similarly

$$
\tilde{g}(z)-\tilde{g}(\hat{z}) \geq-x(z-\hat{z}) .
$$

Let $D_{1}, D_{2} \subset Z$ be bounded domains such that $\sigma \subset D_{1} \subset D_{2} \subset \subset \gamma$. Next, let $l(z) \in C^{\infty}\left(\mathbf{R}^{k}\right)$ be supported on the unit ball $B_{1} \subset \mathbf{R}^{k}$ and $\int_{\mathbf{R}^{k}} l(z) d z=1$ and set

$$
l_{\delta}(z)=\frac{1}{\delta^{k}} l\left(\frac{z}{\delta}\right) .
$$

Let $h \in C^{\infty}(Z), h=1$ on $D_{1}, h=0$ on $Z \backslash D_{2}, 0 \leq h \leq 1$ on $Z$. Set

$$
\begin{gathered}
g_{\epsilon}=\widetilde{g} * l_{\epsilon}, \\
G_{\epsilon}=h g+(1-h) g_{\epsilon} .
\end{gathered}
$$

Since the graph of the function $g$ satisfies the $\lambda$-cone condition it follows that the upper normals to the graph is in the cone $K$. Since $K^{*}$ is a convex cone the upper normals to the graphs of the functions $g_{\epsilon}$ satisfies the $2 \lambda$-cone condition for all small $\epsilon>0$, and hence the graphs of linear function $d_{z} g_{\epsilon}$ is in $L_{2 \lambda}$ for all $z$ where $d_{z}$ is the differential at $z$. Since the functions $g_{\epsilon}$ are defined on the whole space $\mathbf{R}^{k}$ it follows that the graphs of the functions $g_{\epsilon}$ satisfies the $\lambda$-cone condition. Really, let $a, b, a \neq b$ be on the graph of $g_{\epsilon}$. If $a-b \notin L_{2 \lambda}$ then there is a point $\alpha \in[\pi(a), \pi(b)]$ such that $d_{\alpha} g_{\epsilon} \notin L_{2 \lambda}$. For any $k>0$ the function $g_{\epsilon} \rightarrow g$ in $C^{k}\left(D_{2}\right)$ as $\epsilon \rightarrow 0$. Hence for a sufficiently small $\epsilon_{o}>0$ the graph of the function $G_{\epsilon_{0}}:=G$ will satisfy the $2 \lambda$-cone condition. Moreover $G$ will be a smooth function on $Z, G=g$ on $D_{1}$ and $|\nabla G|<\sqrt{n}$ on $Z$.

Let us set

$$
F:=s-G(z) .
$$

Denote

$$
\begin{aligned}
b & :=\nabla F=(-\nabla G, 1), \\
a & :=(\nabla G /|\nabla G|,|\nabla G|) .
\end{aligned}
$$


The vector $a$ is tangent to the level surface of the function $F$, and $\operatorname{tr}(b)=\sqrt{n}$. Since level surfaces of the function $F$ satisfies the $2 \lambda$-cone condition and $a \cdot b=0$, it follows that $a \in L_{2 \lambda}$ and hence $b \in K_{2 \lambda}$. Therefore the function $F$ satisfies the ellipticity conditions with the ellipticity constant $\Lambda<4 \lambda^{2} \sqrt{n}$.

Remark 1. For a real-analytic manifold $\Sigma$ one can obtain the existence of a real-analytic function $F$ after insignificant changes in the construction.

Remark 2. The proof of the lemma holds if instead of compactness of $\Sigma$ we assume that $\Sigma$ is a smooth closed manifold with a boundary.

Proof of Main Lemma. Set

$$
\lambda=(n-1) M
$$

Let $\xi$ and $\eta$ be correspondingly negative and nonnegative subspace of the quadratic form $a-b$ in $\mathbf{R}^{n}$. Denote by $c \in Q$ the quadratic form $l|\xi|^{2}+m|\eta|^{2}, l, m>0$ such that $(a-b) \cdot c=0$. Then $1 /(n-1) M<l / m<(n-1) M$ by (H) 2) and hence the set $H\left(S^{n-1}\right)$ satisfies the $\lambda$-cone condition.

For $\Sigma=H\left(S^{n-1}\right)$ we define function $F$ by Lemma. Then the function $w$ satisfies the equation

$$
F\left(D^{2} w\right)=0
$$

on $\mathbf{R}^{n} \backslash\{0\}$. We show now that $w$ is a viscosity solution of (1) on the whole space $\mathbf{R}^{n}$.

Let $p(x), x \in \mathbf{R}^{n}$ be a quadratic form such that $p \leq w$ on $\mathbf{R}^{n}$. We choose any quadratic form $p^{\prime}(x)$ such that $p \leq p^{\prime} \leq w$ and there is a point $x^{\prime} \neq 0$ at which $p^{\prime}\left(x^{\prime}\right)=w\left(x^{\prime}\right)$. Then it follows that $F(p) \leq F\left(p^{\prime}\right) \leq 0$. Consequently for any quadratic form $p(x)$ from the inequality $p \leq w(p \geq w)$ it follows that $F(p) \leq 0(F(p) \geq 0)$. This implies that $w$ is a viscosity solution of (1) in $\mathbf{R}^{n}$ (see Proposition 2.4 in $[\mathrm{CC}]$ ).

\section{Cubic form $P$}

In this section we introduce and investigate the cubic form which will be used to construct our non-classical solutions. Let $V=(X, Y, Z) \in \mathbf{R}^{12}$ be a variable vector with $X, Y$, and $Z \in \mathbf{R}^{4}$. For any $t=\left(t_{0}, t_{1}, t_{2}, t_{3}\right) \in \mathbf{R}^{4}$ we denote by $q t=t_{0}+t_{1} \cdot i+t_{2} \cdot j+t_{3} \cdot k \in \mathbf{H}$ (Hamilton quaternions). For any $q=$ $q_{0}+q_{1} \cdot i+q_{2} \cdot j+q_{3} \cdot k \in \mathbf{H}$ its conjugate will be denoted $q^{*}=q_{0}-q_{1} \cdot i-q_{2} \cdot j-q_{3} \cdot k$; thus, $q^{*} q=q q^{*}=|q|^{2}=q_{0}^{2}+q_{1}^{2}+q_{2}^{2}+q_{3}^{2}$.

Define the cubic form $P=P(V)=P(X, Y, Z)$ as follows

$$
\begin{gathered}
P(X, Y, Z)=R e(q X \cdot q Y \cdot q Z)=X_{0} Y_{0} Z_{0}-X_{0} Y_{1} Z_{1}-X_{0} Y_{2} Z_{2}-X_{0} Y_{3} Z_{3} \\
-X_{1} Y_{0} Z_{1}-X_{1} Y_{1} Z_{0}-X_{1} Y_{2} Z_{3}+X_{1} Y_{3} Z_{2}-X_{2} Y_{0} Z_{2}+X_{2} Y_{1} Z_{3}-X_{2} Y_{2} Z_{0}-X_{2} Y_{3} Z_{1}
\end{gathered}
$$




$$
-X_{3} Y_{0} Z_{3}-X_{3} Y_{1} Z_{2}+X_{3} Y_{2} Z_{1}-X_{3} Y_{3} Z_{0} .
$$

Let $d=(a, b, c) \in \mathbf{R}^{12}$ be a vector with the norm $\sqrt{3}$, $\|a\|^{2}+\|b\|^{2}+\|c\|^{2}=3$. Define the quadratic form

$$
Q_{d}=Q_{a, b, c}=Q_{a, b, c}(X, Y, Z)
$$

by differentiating $P$ in the direction $d$ :

$$
Q_{a, b, c}(X, Y, Z)=\sum_{i=0}^{4} a_{i} \partial P / \partial X_{i}+\sum_{i=0}^{4} b_{i} \partial P / \partial Y_{i}+\sum_{i=0}^{4} c_{i} \partial P / \partial Z_{i} .
$$

A direct calculation shows that

$$
Q_{d}(X, Y, Z)=X^{t} M_{c} Y+X^{t} M_{b}^{t} Z+Y^{t} M_{a} Z
$$

where, in general, we define the matrix $M_{s}$ for an arbitrary $s \in \mathbf{R}^{4}$ by

$$
M_{s}=\left(\begin{array}{cccc}
s_{0} & -s_{1} & -s_{2} & -s_{3} \\
-s_{1} & -s_{0} & -s_{3} & s_{2} \\
-s_{2} & s_{3} & -s_{0} & -s_{1} \\
-s_{3} & -s_{2} & s_{1} & -s_{0}
\end{array}\right)
$$

Direct (and easy) calculations show that $M_{s}$ has the following properties:

1). $\quad M_{s} \cdot M_{s}^{t}=M_{s}^{t} \cdot M_{s}=\|s\|^{2} I_{4}$ thus, $M_{s}$ is proportional to an orthogonal matrix. In particular, if $\|s\|=1$ then $M_{s}$ is orthogonal itself. In general, we write $M_{s}=\|s\| O_{s}$ with $O_{s} \in O(4)$.

$2) \cdot \operatorname{det}\left(M_{s}\right)=-\|s\|^{4}, \quad \operatorname{det}\left(O_{s}\right)=-1$;

$3)$. the characteristic polynomial $P M_{s}(x)$ of $M_{s}$ factors as

$$
P M_{s}(x)=\left(x^{2}-\|s\|^{2}\right)\left(x^{2}+2 s_{0} x+\|s\|^{2}\right)
$$

with $s_{0}=\operatorname{Re}(q s)$; and that of $O_{s}$ as

$$
P O_{s}(x)=\left(x^{2}-1\right)\left(x^{2}+2 s_{0}^{*} x+1\right)
$$

with $s_{0}^{*}=s_{0} /|q s|=\operatorname{Re}(q s /|q s|)$;

4). define the symmetric matrix $N_{s}=\left(O_{s}+O_{s}^{t}\right)$; then its characteristic polynomial $P N_{s}(x)=\left(x^{2}-4\right)\left(x+2 s_{0}^{*}\right)^{2}$, its spectrum being $S p\left(N_{s}\right)=\left\{2,-2,-2 s_{0}^{*},-2 s_{0}^{*}\right\}$;

5). $M_{s}$ is the matrix (with respect to the standard basis) of the endomorphism $\mathbf{H} \rightarrow \mathbf{H}, q \mapsto \bar{q} \cdot \bar{q} s$. 
The points 3 and 5 applied to the product matrix $M_{r s t}=M_{r} \cdot M_{s} \cdot M_{t}, r, s, t$ being arbitrary vectors in $\mathbf{R}^{4}$ give the following formula for the characteristic polynomial $P M_{r s t}$ of $M_{r s t}$ :

$$
P M_{r s t}(x)=\left(x^{2}-\|r\|^{2}\|s\|^{2}\|t\|^{2}\right)\left(x^{2}+2 P(r, s, t) x+\|r\|^{2}\|s\|^{2}\|t\|^{2}\right)
$$

with $P(r, s, t)=\operatorname{Re}(q r \cdot q s \cdot q t)$ as above. Indeed, $M_{r s t}$ is conjugate to the matrix of the endomorphism $q \mapsto \bar{q} \cdot \bar{q} r \cdot \bar{q} s \cdot \bar{q} t$.

For the corresponding orthogonal matrix $O_{r s t}$ we get the polynomial

$$
P O_{r s t}(x)=\left(x^{2}-1\right)\left(x^{2}+2 \bar{P}(r, s, t) x+1\right)
$$

where $\bar{P}(r, s, t)=P(r, s, t) /(\|r\| \cdot\|s\| \cdot\|t\|)$ and for the corresponding symmetric matrix $N_{r s t}=O_{r s t}+O_{r s t}^{t}$ the spectrum is

$$
(*) \quad S p\left(N_{r s t}\right)=\{2,-2,-2 \bar{P}(r, s, t),-2 \bar{P}(r, s, t)\} .
$$

Warning: in the case of the product of two matrices $M_{r}, M_{s}$ the characteristic polynomial is completely different; namely, if $M_{r s}=M_{r} \cdot M_{s}$ then

$$
P M_{r s}(x)=\left(x^{2}-2(r, s) x+\|r\|^{2}\|s\|^{2}\right)^{2}
$$

with the usual scalar product $(r, s)$.

Define now two quantities $m=m(d)=m(a, b, c)=\|a\| \cdot\|b\| \cdot\|c\|$, $n=n(d)=n(a, b, c)=P(a, b, c)$. Clearly, $|n(d)| \leq m(d) \leq 1$ by the inequality between the geometric and quadratic means, since $\|a\|^{2}+\|b\|^{2}+\|c\|^{2}=3$.

Proposition 1. The characteristic polynomial $\mathrm{CH}_{d}(x)$ of the quadratic form $2 Q_{d}$ equals

$$
C H_{d}(x)=\left(x^{3}-3 x+2 m\right)\left(x^{3}-3 x-2 m\right)\left(x^{3}-3 x+2 n\right)^{2}
$$

Proof. We have

$$
\begin{aligned}
& Q_{d}(X, Y, Z)=X^{t} M_{c} Y+X^{t} M_{b}^{t} Z+Y^{t} M_{a} Z \\
= & \|c\| X^{t} O_{c} Y+\|b\| X^{t} O_{b}^{t} Z+\|a\| Y^{t} O_{a} Z .
\end{aligned}
$$

Let us perform the orthogonal change of variables given by:

$$
x=O_{c}^{t} X, \quad y=Y, \quad z=O_{a} Z .
$$

Then in these new variables the form $Q_{d}$ becomes equal to

$$
\tilde{Q}_{d}(x, y, z)=\|c\| x^{t} y+\|b\| x^{t} O_{c}^{t} \cdot O_{b}^{t} \cdot O_{a}^{t} z+\|a\| y^{t} z .
$$


Thus, the matrix of the form $2 \tilde{Q}_{d}$ is the following block matrix:

$$
\tilde{M}_{d}=\left(\begin{array}{ccc}
0_{4} & \|c\| I_{4} & \|b\| O_{a b c}^{t} \\
\|c\| I_{4} & 0_{4} & \|a\| I_{4} \\
\|b\| O_{a b c} & \|a\| I_{4} & 0_{4}
\end{array}\right)
$$

where $0_{4}$ and $I_{4}$ are the zero and the unit $4 \times 4$ matrices, respectively, $O_{a b c}=O_{a} \cdot O_{b} \cdot O_{c}$ as above.

Let now $\lambda \in S p\left(\tilde{M}_{d}\right), v_{\lambda}=\left(x_{\lambda}, y_{\lambda}, z_{\lambda}\right)$ being a corresponding eigenvector, normalized by the conditions $\left\|v_{\lambda}\right\|=\sqrt{3},\left(v_{\lambda}, d\right) \geq 0$.

The condition $\tilde{M}_{d} \cdot v_{\lambda}=\lambda v_{\lambda}$ gives

$$
\begin{aligned}
& \lambda x_{\lambda}=\|c\| y_{\lambda}+\|b\| O_{a b c}^{t} z_{\lambda} \\
& \lambda y_{\lambda}=\|c\| x_{\lambda}+\|a\| z_{\lambda} \\
& \lambda z_{\lambda}=\|b\| O_{a b c} x_{\lambda}+\|a\| y_{\lambda}
\end{aligned}
$$

Multiplying the second and the third equations by $\lambda$ and inserting in thus obtained equations the first one one finds

$$
\begin{aligned}
& \left(\lambda^{2}-\|c\|^{2}\right) x_{\lambda}=\left(\|a\| \cdot\|c\|+\lambda\|b\| O_{a b c}^{t}\right) z_{\lambda} \\
& \left(\lambda^{2}-\|a\|^{2}\right) z_{\lambda}=\left(\|a\| \cdot\|c\|+\lambda\|b\| O_{a b c}\right) x_{\lambda}
\end{aligned}
$$

which implies

$$
\left(\lambda^{2}-\|a\|^{2}\right)\left(\lambda^{2}-\|c\|^{2}\right) x_{\lambda}=\left(\|a\| \cdot\|c\|+\lambda\|b\| O_{a b c}^{t}\right)\left(\|a\| \cdot\|c\|+\lambda\|b\| O_{a b c}\right) x_{\lambda}
$$

and, after simplifying,

$$
\lambda\left(\lambda^{3} I_{4}-3 \lambda I_{4}-m N_{a b c}\right) x_{\lambda}=0,
$$

since $\|a\|^{2}+\|b\|^{2}+\|c\|^{2}=3, m=\|a\| \cdot\|b\| \cdot\|c\|, O_{a b c} O_{a b c}^{t}=I_{4}$, $N_{a b c}=O_{a b c}+O_{a b c}^{t}$.

Hence, either $\lambda=0$ or

$$
\left(\lambda^{3}-3 \lambda\right) \in m \cdot S p\left(N_{a b c}\right)=\{-2 m, 2 m,-2 n,-2 n\} .
$$

This finishes the proof for $\lambda \neq 0$. If $\lambda=0$ we get the conditions

$$
\begin{aligned}
& 0=\|c\| y_{\lambda}+\|b\| O_{a b c}^{t} z_{\lambda} \\
& 0=\|c\| x_{\lambda}+\|a\| z_{\lambda} \\
& 0=\|b\| O_{a b c} x_{\lambda}+\|a\| y_{\lambda}
\end{aligned}
$$

immediately implying that $m=0$ (since else these equations give $x_{\lambda}=0$ ) and the formula holds for this case as well. 
Corollary 1. Define the angles $\alpha, \beta \in[0, \pi]$ by $m=\cos \alpha, n=\cos \beta$. Then

$S p\left(\tilde{M}_{d}\right)=\{2 \cos (\alpha / 3+\pi k / 3), 2 \cos (\beta / 3+\pi(2 l+1) / 3), 2 \cos (\beta / 3+\pi(2 l+1) / 3)\}$, $k=0,1, \ldots 5, \quad l=0,1,2$.

Proof. Indeed, if we put $\lambda=2 \cos \gamma$, the equations $\lambda^{3}-3 \lambda+2 m=0$, $\lambda^{3}-3 \lambda-2 m=0$ and $\lambda^{3}-3 \lambda+2 n=0$ become respectively, $\cos (3 \gamma)=-\cos \alpha$, $\cos (3 \gamma)=\cos \alpha$ and $\cos (3 \gamma)=-\cos \beta$ which implies the result.

Let us now order the eigenvalues in the decreasing order:

$$
\lambda_{1} \geq \lambda_{2} \geq \lambda_{3} \geq \lambda_{4} \geq \lambda_{5} \geq \lambda_{6} \geq \lambda_{7} \geq \lambda_{8} \geq \lambda_{9} \geq \lambda_{10} \geq \lambda_{11} \geq \lambda_{12} .
$$

\section{Corollary 2.}

1). $2 \geq \lambda_{1} \geq \lambda_{2} \geq \lambda_{3} \geq \lambda_{4} \geq 1$

2). $-1 \geq \lambda_{9} \geq \lambda_{10} \geq \lambda_{11} \geq \lambda_{12} \geq-2$;

3). $\lambda_{1} \geq \sqrt{3} ; \lambda_{12} \leq-\sqrt{3}$;

4). If $\lambda_{1} / \lambda_{3}=2$ (resp. $\left.\lambda_{12} / \lambda_{10}=2\right)$ then the polynomial

$C H_{d}(x)=(x+2)^{3}(x-2)(x+1)^{2}(x-1)^{6}\left(\right.$ resp. $C H_{d}(x)=(x-2)^{3}(x+2)(x-$ $\left.1)^{2}(x+1)^{6}\right)$, and $d=v_{1}$ (resp. $\left.d=v_{12}\right)$ where $v_{i}$ is the normalized eigenvector corresponding to $\lambda_{i}$.

Proof. All these conclusions, except that concerning $v_{1}$ (resp. $v_{12}$ ) follow from Corollary 1 along with the following elementary lemma:

Lemma. Let $F_{m}(x)=\left(x^{3}-3 x-2 m\right)$ with $|m| \leq 1$, and let $x_{1} \geq x_{2} \geq x_{3}$ be its roots.

1). If $0 \leq m \leq 1$ then $2 \geq x_{1} \geq \sqrt{3},-1 \geq x_{3}$, and each of the conditions $2=x_{1},-1=x_{3}$ implies $m=1$.

2). If $0 \geq m \geq-1$ then $-2 \leq x_{3} \leq-\sqrt{3}, 1 \leq x_{1}$, and each of the conditions $1=x_{1},-2=x_{3}$ implies $m=-1$.

3). If $|m| \leq 0.75$ then $\left|x_{1}\right|>1.38,\left|x_{3}\right|>1.38$.

This lemma follows from the monotonicity of $\cos (x)$ on $[0, \pi]$ along with the inequalities $2 \cos \left(\frac{\arccos (.75)+2 \pi}{3}\right)<-1.38,2 \cos \left(\frac{\arccos (.75)}{3}\right)>1.94$.

To prove that $d=v_{1}$ one notes that $\lambda_{1} / \lambda_{3}=2$ implies $m(d)=n(d)=1$ which means the function $P$ has an absolute maximum at $d$, its derivative in the direction $d$ equals 1 which means that $2 Q_{d}(d)=2$, i.e. $d=v_{1}$. The case of $v_{12}$ is completely similar.

Corollary 3. Define

$$
\delta=\sup _{d,\|d\|=\sqrt{3}}\left\{\max \left\{\frac{\lambda_{+}^{\perp}(d)}{\lambda_{3}(d)}, \quad \frac{\lambda_{-}^{\perp}(d)}{\lambda_{10}(d)}\right\}\right\}
$$


where

$$
\lambda_{+}^{\perp}(d)=2 \sup _{v \perp d,\|v\|=\sqrt{3}} Q_{d}(v), \quad \lambda_{-}^{\perp}(d)=2 \inf _{v \perp d,\|v\|=\sqrt{3}} Q_{d}(v) .
$$

Then $\delta<3 / 2$.

Proof. By Lemma, part 3 it is true for $|n| \leq 0.75$ since $2 / 1.38<3 / 2$. Let now $n \geq 0.75$ (the case $n \leq-0.75$ being symmetric). Suppose that $\frac{\lambda_{+}^{\perp}(d)}{\lambda_{3}(d)} \geq 3 / 2$, and hence $\lambda_{+}^{\perp}(d) \geq 1.5$ (since $\lambda_{3}(d) \geq 1$ ). We will show that the conditions $\lambda_{+}^{\perp}(d) \geq 1.5$ and $n \geq 0.75$ are incompatible. Indeed, define

$$
T(x, y)=P\left(x d+y \sqrt{3} v_{+}^{\perp}(d)\right)=t_{3} x^{3}+t_{2} x^{2} y+t_{1} x y^{2}+t_{0} y^{3}
$$

where $v_{+}^{\perp}(d)$ is a norm $\sqrt{3}$ vector on which $Q_{d}(v)$ achieves the maximum. We get that $t_{3}=T(1,0)=P(d)=n \geq 0.75, t_{1}=T_{x}(0,1)=3 \lambda_{+}^{\perp}(d) / 2 \geq 9 / 4$. For any $(x, y)$ with $x^{2}+y^{2}=1,|T(x, y)|=\left|P\left(x d+y \sqrt{3} v_{+}^{\perp}(d)\right)\right| \leq 1$. Let now $x_{0}=\frac{1}{\sqrt{2}}, y_{0}= \pm \frac{1}{\sqrt{2}}$ where the sign of $y_{0}$ is chosen from the condition that $y_{0} \frac{t_{0}+t_{2}}{\sqrt{2}} \geq 0$. Then $\left|T\left(x_{0}, y_{0}\right)\right| \geq \frac{t_{3}+t_{1}}{2 \sqrt{2}} \geq \frac{3}{2 \sqrt{2}}>1$ which is a contradiction.

The following result will be used in Section 4 to deduce our main result.

Corollary 4. Let $u \neq v \in S_{\sqrt{3}}^{11}$ be two vectors of norm $\sqrt{3}$. Then

$$
\begin{aligned}
& P(u)-P(v) \leq 3 \sqrt{3} \lambda_{3}(d)|u-v| / 4 . \\
& P(u)-P(v) \geq 3 \sqrt{3} \lambda_{10}(d)|u-v| / 4 .
\end{aligned}
$$

where $d=\sqrt{3} \frac{u-v}{|u-v|}$.

Proof. Denote $s=|u-v| / 2, z=d^{\perp} \bigcap[u, v]$. Writing the Taylor development for the (cubic) function $P$, we get

$$
P(u)-P(v)=2 s\left(Q_{d}(z) / \sqrt{3}+s^{2} P_{d d d} / 18 \sqrt{3}\right) .
$$

Since

$$
\begin{aligned}
& Q_{d}(z) \leq\left(1-s^{2} / 3\right) \sup _{x \perp d,\|x\|=\sqrt{3}} Q_{d}(x) \leq 3\left(1-s^{2} / 3\right) \lambda_{+}^{\perp}(d) / 2 \leq 9\left(1-s^{2} / 3\right) \lambda_{3}(d) / 4, \\
& P_{d d d} \leq 2 / \sqrt{3} \leq 2 \lambda_{3}(d) / \sqrt{3} \text { we get } \\
& \quad P(u)-P(v) \leq 2 s \lambda_{3}(d)\left(9\left(1-s^{2} / 3\right) / 4 \sqrt{3}+s^{2} / 27\right) \leq 3 \sqrt{3} \lambda_{3}(d)|u-v| / 4 .
\end{aligned}
$$

The proof of the second inequality is completely similar.

Remark. Let us resume the spectral properties of $2 Q_{d}$ when $d$ varies over $S=S_{\sqrt{3}}^{11}$. We have a stratification $V_{0} \subset S \supset T \supset V=V_{+} \cup V_{-}$where $T=$ 
$S_{1}^{3} \times S_{1}^{3} \times S_{1}^{3}$ is defined by the condition $m(d)=1, V_{+}$(resp. $V_{-}$) is defined by $P(d)=1$ (resp. $P(d)=-1$ ), $V_{0}=\{d: m(d)=0\}$; each of $V_{+}$and $V_{-}$is diffeomorphic to $S_{1}^{3} \times S_{1}^{3}$. On $V_{+}$(resp. $V_{-}$) we have the characteristic polynomial $(x+2)^{3}(x-2)(x+1)^{2}(x-1)^{6}\left(\operatorname{resp} .(x-2)^{3}(x+2)(x-1)^{2}(x+1)^{6}\right)$; on $S \backslash\left(T \cup V_{0}\right)$ we have $\sqrt{3}<\lambda_{1}(d)<2,1<\lambda_{4}(d),-1>\lambda_{9}(d),-\sqrt{3}>$ $\lambda_{12}(d)>-2$. Finally, on $V_{0}$ the polynomial equals $x^{4}\left(x^{2}-3\right)^{4}$.

\section{Function w and map $\mathrm{H}$}

In this section we show that the function

$$
w(x)=P(x) /|x|
$$

is what we want, i. e. the map

$$
H: S_{1}^{11} \longrightarrow Q, \quad H(a)=\operatorname{Hess}(w(a))
$$

verifies the condition $(\mathrm{H})$ of Section 2 .

Proposition 2. Let $a \neq b \in S_{1}^{11}$. Then there exist two vectors $e, f \in S_{1}^{11}$ such that

$$
\begin{gathered}
w_{e e}(a)-w_{e e}(b) \geq|a-b| / 4 \sqrt{3}, \\
w_{f f}(a)-w_{f f}(b) \leq-|a-b| / 4 \sqrt{3} .
\end{gathered}
$$

Proof. Let $d=\sqrt{3} \frac{a-b}{|a-b|}$. Recall that we denote by $v_{i}, i=1, \ldots, 12$ the normalized eigenvectors of the form $2 Q_{d}$ from Section 3 (the eigenvalues $\lambda_{i}=$ $\lambda_{i}(d)$ being ordered in the decreasing order). Let $V^{+}$be the 3 -dimensional space generated by $v_{1}, v_{2}, v_{3}$ and let $e \in S_{1}^{11} \cap V^{+} \bigcap a^{\perp} \bigcap b^{\perp}$. It means in particular that $2 Q_{d}(e) \geq \lambda_{3}(d)$. The conditions $b \perp e, a \perp e$ imply

$$
w_{e e}(a)=P_{e e}(a)-P(a), w_{e e}(b)=P_{e e}(b)-P(b),
$$

hence

$$
w_{e e}(a)-w_{e e}(b)=P_{e e}(a)-P_{e e}(b)-(P(a)-P(b)) .
$$

Since $P_{e e}(x)$ is a linear function we get

$P_{e e}(a)-P_{e e}(b)=|a-b| P_{e e d} / \sqrt{3}=2|a-b| Q_{d}(e) / \sqrt{3} \geq \lambda_{3}(d)|a-b| / \sqrt{3}$.

Now,

$P(a)-P(b)=\frac{(P(a \sqrt{3})-P(b \sqrt{3}))}{3 \sqrt{3}} \leq \frac{3 \sqrt{3} \lambda_{3}(d) \sqrt{3}|a-b|}{12 \sqrt{3}}=\frac{\sqrt{3} \lambda_{3}(d)|a-b|}{4}$ 
by Corollary 4, and we get

$$
w_{e e}(a)-w_{e e}(b) \geq \lambda_{3}(d)|a-b|\left(\frac{1}{\sqrt{3}}-\frac{\sqrt{3}}{4}\right) \geq \frac{|a-b|}{4 \sqrt{3}} .
$$

The second inequality is proven replacing $e$ by $f \in S^{11} \cap V^{-} \cap a^{\perp} \cap b^{\perp}$ where $V^{-}$is generated by $v_{10}, v_{11}, v_{12}$.

\section{Corollary 5.}

1). The map $H: S_{1}^{11} \longrightarrow Q, \quad H(a)=H e s s(w(a))$ is a smooth embedding.

2). Let for $a \neq b \in S_{1}^{11}$

$$
\mu_{1} \geq \mu_{2} \geq \ldots \geq \mu_{11} \geq \mu_{12}
$$

be the eigenvalues of Hess $(w(a))-H e s s(w(b))$. Then

$$
M^{-1}=\frac{1}{1536 \sqrt{3}} \leq-\frac{\mu_{1}}{\mu_{12}} \leq 1536 \sqrt{3}=M .
$$

Proof.

1). This follows immediately from Proposition 2 .

$2)$. An easy calculation shows that $\left|w_{e f g}(x)\right| \leq 32$ for any $e, f, g, x \in S_{1}^{11}$. Hence

$$
\left|w_{e f}(a)-w_{e f}(b)\right| \leq\left|w_{e f d^{\prime}}\left(d^{\prime}\right)\right| \cdot|a-b| \leq 32|a-b|
$$

for $d^{\prime}=d / \sqrt{3}$. Since all elements of the matrix Hess $(w(a))-\operatorname{Hess}(w(b))$ are of absolute value $\leq 32|a-b|$, all its eigenvalues are of absolute value $\leq 12 \cdot 32|a-b|$. Using the inequalities of Proposition 2 we get the conclusion.

\section{REFERENCES}

[A] A.D. Alexandrroff; Sur les théorèmes d'unicite pour les surfaces fermées, Dokl. Acad. Nauk 22 (1939), 99-102.

[CC] L. Caffarelli, X. Cabré; Fully Nonlinear Elliptic Equations, Amer. Math. Soc., Providence, R.I., 1995.

[CIL] M.G. Crandall, H. Ishii, P-L. Lions; User's guide to viscosity solutions of second order partial differential equations, Bull. Amer. Math. Soc. (N.S.) 27(1) (1992), 1-67.

[GT] D. Gilbarg, N. Trudinger; Elliptic Partial Differential Equations of Second Order, 2nd ed., Springer-Verlag, Berlin-Heidelberg-New York-Tokyo, 1983. 
[HNY] Q. Han, N. Nadirashvili, Y. Yuan, Linearity of homogeneous orderone solutions to elliptic equations in dimension three, Comm. Pure Appl. Math. 56 (2003), 425-432

[K] N.V. Krylov; Nonlinear Elliptic and Parabolic Equations of Second Order, Reidel, 1987.

[NY] N. Nadirashvili, Y. Yuan, Homogeneous solutions to fully nonlinear elliptic equation, Proc. AMS 134 (2006), no 6.

[N] L. Nirenberg; On Nonlinear Elliptic Partial Differential Equations and Hölder Continuity, Comm. Pure Appl. Math. 6 (1953), 103-156. 